\title{
Os hotéis como pólos geradores de viagens
}

\author{
Lenise Grando Goldner'; Diego Westphal'; \\ Jorge Augusto Martins Gonçalves ${ }^{3}$; Ronaldo Balassiano ${ }^{4}$
}

\begin{abstract}
Resumo: Os estudos sobre hotéis analisados como Pólos Geradores de Viagens são escassos tanto no Brasil quanto no exterior. Neste artigo desenvolve-se uma análise e compilação de alguns trabalhos realizados nos EUA sobre o tema, com destaque para os modelos do ITE- Institute of Transportation Engineers - para diferentes categorias de hotéis. Para o Brasil são analisados os estudos de Feitosa (2003) e de Goldner et al (2006) sobre os padrões de viagens, bem como os de Goldner e Inocêncio (2007) sobre taxas e modelos de geração de viagens para os hotéis da cidade de Florianópolis. Uma análise comparativa entre os principais modelos é realizada, buscando verificar a adequação dos modelos do ITE à realidade brasileira. Os resultados ratificam a importância do desenvolvimento de novos modelos com base em dados que caracterizam a especificidade da geração de viagens no caso do Brasil.
\end{abstract}

\begin{abstract}
Studies analyzing hotels and their role as trip generation hubs are rare in Brazil and abroad. In this paper, an analysis and compilation of some work linked to the subject, developed in the USA, is carried out, with emphasis on the ITE - Institute of Transportation Engineers - models, to different hotel categories. In the case of Brazil, the studies of Feitosa (2003) and Goldner et al. (2006) assessing trip patterns and also those of Goldner and Inocencio (2007), who analyzed rates and trip generation models to hotels located in Florianopolis. A comparative analysis among the main models is also carried out, aiming at the verification of the adequacy of ITE models to the Brazilian reality. The comparison ratifies the importance to develop models based on data that characterize the specificity of trip generation in the case of Brazil.
\end{abstract}

\section{INTRODUÇÃO}

Os Pólos Geradores de Viagens (PGV's) têm sido estudados exaustivamente em diferentes países. Dentre os mais conhecidos através da bibliografia internacional encontram-se os shopping centers e os supermercados, para os quais existem inúmeros estudos, para realidades de diferentes países, tendo como base a experiência americana (Keefer,1966; ITE, 1991, 1997, 2001, 2008; USDOT, 1995; Peyrebrune, 1996; Kikuchi et al, 2006), bem como modelos e parâmetros para o Brasil (Conceição, 1984; Grando, 1986; Silveira, 1990; Goldner,1994; Portugal e Goldner, 2003; Della Giustina, 2005; Silva, 2006, Amâncio e Guimarães, 2007; Freitas e Raia Jr., 2008; Souza et al, 2009), e demais países da América Latina, cujas bibliografias podem ser obtidas no portal da Rede Ibero-Americana de Estudos em Pólos Geradores de Viagens (REDPGV) na internet, através do link "produção da rede".

Estudos sobre aeroportos brasileiros sob a ótica da

\footnotetext{
${ }^{1}$ Lenise Grando Goldner, Departamento de Engenharia Civil, Universidade Federal de Santa Catarina, Florianópolis, SC, Brasil. (e-mail: lenise@ecv.ufsc.br).

${ }^{2}$ Diego Westphal, Departamento de Engenharia Civil, Universidade Federal de Santa Catarina, Florianópolis, SC, Brasil. (e-mail: diegow@matrix.com.br).

${ }^{3}$ Jorge Augusto Martins Gonçalves, Programa de Engenharia de Transportes, Universidade Federal do Rio de Janeiro, Rio de Janeiro, RJ, Brasil. (e-mail: goncalves@pet.coppe.ufrj.br).

${ }^{4}$ Ronaldo Balassiano, Programa de Engenharia de Transportes, Universidade Federal do Rio de Janeiro, Rio de Janeiro, RJ, Brasil. (e-mail: ronaldo@pet.coppe.ufr.br).
}

Manuscrito recebido em 22/2/2010 e aprovado para publicação em 17/3/2010. Este artigo é parte de TRANSPORTES, volume XVIII, número 1, março de 2010. ISSN: 2237-1346 (online). engenharia de tráfego, foram também desenvolvidos (Goldner et al, 2005), e iniciaram-se os estudos de hotéis no Brasil (Feitosa e Balassiano, 2003; Goldner et al, 2006; Goldner e Inocêncio,2007), bem como de instituições de ensino superior (Nunes, 2005; Souza, 2007) e de ensino médio (Bertazzo, 2008), entre vários outros PGV's estudados.

Considerando-se que os hotéis têm sido pouco estudados, o objetivo principal deste trabalho é analisar esse tipo de edificação como Pólo Gerador de Viagens. A análise parte de uma revisão bibliográfica sobre os principais modelos de geração de viagens desenvolvidos no exterior e no Brasil para diferentes categorias de hotéis. Após uma avaliação comparativa desses modelos, são considerados tanto os aspectos metodológicos utilizados quanto os aspectos quantitativos, analisando os resultados produzidos pelos diferentes métodos. Complementarmente ao estudo serão descritos e comparados os padrões de viagens para os hotéis, para casos específicos encontrados na bibliografia.

A compilação de dados e informações de estudos que avaliam os hotéis, sob a ótica da Engenharia de Tráfego, permite que o planejador possa definir de forma mais fundamentada o método a ser utilizado em cada caso específico. Da mesma forma, os parâmetros e modelos utilizados, principalmente quando se trata da avaliação de impactos gerados pelos hotéis sobre o sistema viário, são mais facilmente identificados. Os métodos utilizados para análise de impacto abrangem várias etapas, sendo uma das mais importantes, a de geração de viagens. Esta é a principal razão para que o trabalho tenha como foco a avaliação dos modelos de 
geração de viagens já desenvolvidos para hotéis.

Espera-se, desta forma, fornecer ao planejador subsídios para uma decisão adequada sobre o modelo a ser adotado, optando pelo que melhor se adapte às necessidades e peculiaridades do empreendimento analisado.

\section{ELEMENTOS DE ANÁLISE}

\subsection{Modelos do ITE}

A principal referência bibliográfica sobre modelos de geração de viagens para hotéis é a publicação do ITE denominada TRIP GENERATION, em suas várias edições (1991, 1997, 2001, 2003 e 2008). Os tipos de hotéis apresentados nesta publicação são: hotéis com ou sem suítes, hotéis de negócios, motéis e resort hotéis.

Para uma melhor compreensão sobre a categoria em que está inserido um hotel que venha a ser estudado, descreve-se a seguir as definições de hotel, tendo como referência a classificação do ITE.

Hotéis: são locais de alojamento que disponibilizam quartos, restaurantes, bares, salas de reunião e de banquete, salas de convenções e lojas. Alguns dos locais incluídos nessa categoria de uso do solo são na atualidade grandes motéis que fornecem os mesmos serviços de um hotel como aqui descrito.

Suites hotels: são locais de alojamento que disponibilizam quartos, um pequeno restaurante e salão, e um pequeno espaço para reuniões. Cada suíte possui sala e quarto separados; normalmente dispõem também de uma pequena cozinha. Esses hotéis são localizados principalmente em áreas de subúrbio.

Business hotels: são locais de alojamento direcionados para o viajante a negócios. Eles disponibilizam quartos e outros serviços limitados como um buffet de café da manha e serviço de bar (não existe serviço de refeições nem área para realização de reuniões ). Cada unidade é composta por um quarto grande para uma pessoa. Nos Estados Unidos eles estão localizados em áreas de subúrbio.

Motéis: são locais de alojamento que disponibilizam quartos, normalmente tem um restaurante, geralmente oferecem estacionamento grátis e raramente dispõem de sala de reuniões.

Resort Hotels: são similares aos hotéis, onde são disponibilizados quartos, restaurantes, bares e lojas. A principal diferença é que os resort hotels são voltados para o turismo e turismo de negócios, normalmente oferecendo uma variedade de serviços de recreação, ao invés de salas de convenções e de reuniões. Resort hotels são normalmente encontrados em áreas de subúrbio ou de periferia e, em geral, são maiores que os hotéis convencionais.

As variáveis consideradas nos modelos de regressão do ITE foram: número de quartos ocupados, número de quartos total e número de empregados. A variável estimada (T) representa o número de viagens horárias aos hotéis, na hora de pico de movimento do hotel, tanto de manhã quanto à tarde, na hora de pico de tráfego do sistema viário adjacente ao hotel, entre 07h00min e $09 \mathrm{~h} 00 \mathrm{~min}$ e entre $16 \mathrm{~h} 00 \mathrm{~min}$ e $18 \mathrm{~h} 00 \mathrm{~min}$.

O estudo apresenta, além dos modelos de regressão simples, as taxas de geração de viagens diárias e horárias para os cenários descritos, bem como as percentagens do fluxo entrando e saindo. Foram elaborados modelos para um dia de semana típico, para o sábado e para o domingo. O número de hotéis por categoria utilizado para a calibração dos modelos de geração de viagens variou para cada caso específico.

\subsection{Modelos de Ackret e Hosea}

O trabalho de Ackret e Hosea (1992) para hotéis cassino de Las Vegas nos EUA, pode ser destacado. O estudo foi desenvolvido com base em uma amostra de 21 hotéis-cassino, agrupados conforme sua localização dentro da cidade de Las Vegas. Os autores elaboraram modelos de acordo com a seguinte distribuição espacial:

Strip Hotels and Casinos (S): - esta categoria representa todas as propriedades localizadas junto à "Las Vegas strip", que é definida como a área junto do Las Vegas Boulevard (extensão total de 8,4 km) entre Sahata Avenue e Sunset Road. Todas as propriedades estão localizadas dentro da área urbana de Las Vegas e são uma atração para os típicos turistas de jogo.

Outlying Áreas (O) - Esta categoria inclui hotéis cassinos rurais, relativamente pequenos, localizados fora da área metropolitana de Las Vegas, principalmente ao longo da via Interstate 15, que liga a área sul da Califórnia à Las Vegas.

Local Casinos (L) - hotéis cassino fora da "Las Vegas strip", com aproximadamente 200 a 300 quartos estão incluídos nesse grupo. Estas propriedades estão geralmente localizadas junto de vias arteriais dentro da área urbana de Las Vegas, e atraem principalmente moradores de Las Vegas.

Os modelos de regressão simples e múltipla foram desenvolvidos para as horas de pico da manhã e da tarde, em função de variáveis como o número de quartos, número de empregados e da área do cassino (1.000 pés quadrados). Os modelos estimam o número de viagens horárias aos cassinos e apresentam também a distribuição direcional do fluxo (entrando e saindo) e as taxas médias de viagens.

\subsection{Modelos de Rowe et al}

Rowe et al (2002) realizaram um aperfeiçoamento dos estudos de Ackret e Hosea (1992) para os hotéiscassino de Las Vegas. A amostra de hotéis pesquisada foi ampliada de 21 para 46 e novos modelos de regressão simples e múltipla foram calibrados. 
Os hotéis-cassino avaliados foram divididos em dois grupos tendo como referência suas localizações. Um hotel-cassino era considerado um "resort corridor" se estivesse localizado dentro da área do Clark County Regional Transportation Commission limitada pelo Valley View Boulevard (oeste), Maryland Parkway (leste), Washington Avenue (norte) e McCarran International Airport (sul - Robendale Road). Estas fronteiras que incluem a famosa Las Vegas "Strip", fornecem os limites do "resort corridor" de Las Vegas.

Um hotel-cassino não localizado no "resort corridor" era classificado como uma instalação local. Essa classificação era necessária para distinguir precisamente as características de geração de viagens desses dois grupos distintos de hotéis-cassino.

Nas instalações de "resort corridor" são geradas várias viagens não-automotivas devido ao fluxo de pedestres que resulta da proximidade dos hotéiscassino. Instalações locais têm um maior volume de viagens automotivas e poucas viagens de pedestres associadas a elas. Isso ocorre pela localização desses resorts estar fora do "resort corridor" e longe de outros hotéis-cassino.

Para os hotéis localizados no "resort corridor", os modelos de regressão linear simples utilizaram as seguintes variáveis: número de posições de jogo, número de empregados, número de quartos do hotel e área de cassino (1000 pés quadrados). Com as mesmas variáveis, foram também calibrados os modelos de regressão múltipla. O número de viagens estimadas considerou separadamente o pico da manhã e o pico da tarde. No primeiro caso a distribuição direcional considerada foi de $59 \%$ do volume entrando na área de influência e de $41 \%$ saindo, enquanto no segundo caso os valores considerados foram de $48 \%$ e $52 \%$ respectivamente.

Para os hotéis locais foram elaborados modelos de regressão linear simples, com as seguintes variáveis: posições de jogo, número de empregados, número de quartos no hotel e área de cassino (1000 pés quadrados). As distribuições direcionais para o pico da manhã foram de $57 \%$ entrando e $43 \%$ saindo e para o pico da tarde de $52 \%$ entrando e $48 \%$ saindo. Para ambas as localizações foram apresentadas as taxas médias de viagem.

\subsection{Estudo de Feitosa}

No Brasil, destaca-se o estudo de Feitosa (2003) sobre os padrões de viagens a hotéis-residência na cidade do Rio de Janeiro. No trabalho realizaram-se entrevistas com os moradores deste tipo de hotel, num total de 83 entrevistas para empreendimentos localizados na zona sul e na Barra da Tijuca. As entrevistas foram realizadas em dias de semana, entre 7:30 e 11:00 horas e
17:30 e 20:00 horas, considerados horários de entrada e saída das edificações. Foram levantados dados sobre o perfil do usuário, hábitos de viagem e hábitos de consumo.

Um formulário complementar foi preenchido através de entrevistas por telefone com os administradores dos hotéis-residência de modo a obter as informações sobre os horários de entrada e saída, o número de vagas de estacionamento disponíveis e os serviços oferecidos.

\subsection{Estudo de Goldner et al}

Goldner et al (2006), realizaram uma pesquisa preliminar em quatro hotéis da cidade de Florianópolis SC, (dois situados no centro e dois nas principais praias), onde se efetuaram entrevistas com os hóspedes e contagens nos estacionamentos e meio-fio de embarque/desembarque. Foram obtidos assim os padrões de viagens a estes hotéis, bem como parâmetros para o dimensionamento dos estacionamentos e da extensão de meio-fio. Devido às flutuações da demanda na cidade, que tem perfil de turismo de verão, levantaram-se as informações em finais de semana e durante a semana, separadamente, nos meses de verão (Janeiro e Fevereiro de 2005) e nos meses típicos (primeira quinzena de dezembro de 2004 e abril de 2005).

As pesquisas foram planejadas de maneira a se obter trinta entrevistas para cada período, resultando em 240 entrevistas no período típico e 240 para o período de pico, totalizando 480 entrevistas. Esta foi a distribuição teórica da amostra, que não se concretizou em alguns casos, devido ao baixo movimento dos hotéis em algumas datas.

Numa segunda etapa deste trabalho, Goldner e Inocêncio (2007) deram continuidade à pesquisa iniciada nos hotéis de Florianópolis, agora com uma amostra ampliada de hotéis da cidade. Em dezesseis hotéis desta cidade, nove localizados no centro e seis nas praias, foram realizados levantamentos dos fluxos de veículos entrando e saindo dos estacionamentos e do meio-fio de embarque/desembarque.

A partir destes dados elaboraram-se taxas de viagens e modelos de geração de viagens, através de regressão linear, relacionando o fluxo entrando e saindo dos acessos com variáveis conhecidas dos hotéis, como o número de quartos, o número de empregados, o número de vagas de estacionamento e a área total construída. Como resultados complementares obtiveram-se também as Percentagens de Pico Horário nos estacionamentos, e o tempo de permanência dos veículos no meio-fio, parâmetros úteis no dimensionamento destas facilidades de transportes.

Para os hotéis do centro, os dados de campo foram levantados no mês de outubro, considerado um mês 
típico, num único dia da semana, uma terça-feira. Nos hotéis da praia os levantamentos foram realizados no mês de fevereiro, considerado um mês de pico de verão, aos sábados, o dia de maior movimento de turistas da semana. As contagens foram realizadas das 7:00 às 19:00 horas, anotando-se a placa dos veículos entrando e saindo do hotel, em intervalos de 15 minutos.

\section{PADRÕES DE VIAGENS}

As conclusões mais importantes sobre o comportamento dos usuários dos hotéis-residência na cidade do Rio de Janeiro, obtidos no estudo de Feitosa (2003) serão apresentados a seguir.

Segunda a autora, uma constatação importante está relacionada ao perfil e aos hábitos de consumo dos moradores deste tipo de PGV. Como as unidades residenciais têm, comparativamente a unidades residenciais convencionais, menor área útil, tendem a abrigar pessoas que residem sozinhas ou são solteiras e pequenas famílias (casais sem filhos), gerando, portanto, um padrão de viagens muito próprio para esses residentes. As unidades não possuem também área específica que permita a guarda de maiores volumes de alimentos e outros produtos, forçando esses residentes à realização de um maior número de viagens para compras.

Embora a atividade mais importante para esses moradores no seu dia-a-dia seja o trabalho, o maior volume de viagens geradas é aquele com motivo "compras", "lazer" e "prática de atividades esportivas". Como essas edificações estão normalmente localizadas em áreas nobres das cidades, onde a oferta de serviços e comércio é bastante diversificada, existe naturalmente uma maior propensão à realização desse tipo de viagem pelos residentes.

Por outro lado, é importante ainda considerar, que a maioria das viagens realizadas, incluindo aquelas que ocorrem nos horários em que a rede viária está mais sobrecarregada, tem o carro particular como principal modo de transporte, contribuindo dessa forma para aumentar os impactos na rede. Ao mesmo tempo, a caminhada é o segundo modo de transporte mais utilizado.

Finalmente verificou-se que um número expressivo das viagens realizadas por esses residentes é de curta extensão (até $5 \mathrm{~km}$ ). A localização desses empreendimentos, o perfil dos seus residentes e as atividades preferidas exercidas no seu cotidiano, concorrem para justificar esse fato.

Considerando que a taxa de propriedade de veículos para esses residentes é elevada e ainda que a utilização do carro particular é intensa, seria importante a elaboração de campanhas de conscientização para um uso mais racional dos sistemas de transportes públicos disponíveis. Cerca de 50\% das viagens realizadas têm extensão inferior a $5 \mathrm{~km}$, o que justificaria um maior incentivo à viagens de bicicleta, ou mesmo a caminhada. Observando-se ainda que cerca de $90 \%$ das viagens realizadas têm extensão inferior a $15 \mathrm{~km}$, o incentivo a maior utilização de sistemas de transporte público poderia ser igualmente recomendável.

Descreve-se a seguir, um resumo das principais conclusões obtidas por Goldner et al (2006) sobre os padrões de viagens dos hóspedes dos hotéis de Florianópolis, em função dos diferentes cenários estudados.

a) $74,63 \%$ das viagens foram exclusivas para o shopping:

- No período típico o motivo principal das viagens foram os negócios, enquanto que no período de pico foram os negócios e o turismo.

- O meio de transporte principal para se chegar à Florianópolis foi o avião, seguido pelo automóvel, com pequena diferença de percentagem entre eles.

- Dentro da cidade as viagens foram feitas principalmente ao centro e às praias durante o dia; à noite visitou-se também o shopping.

- O principal meio de transporte utilizado nestas viagens foi o automóvel, seguido por passeios a pé.

b) Acerca dos hotéis localizados nas praias:

- No período típico os motivos principais das vindas à Florianópolis foram os negócios, convenções e turismo, enquanto no período de pico predominantemente turismo.

- Para se chegar à cidade utilizou-se principalmente o avião, seguido pelo automóvel.

- No período típico os hóspedes permaneceram no hotel durante o dia (especialmente os vindos para convenções) ou visitaram as praias; no período de pico os passeios foram feitos principalmente à praia de dia, e à noite aos bares e restaurantes da orla.

- Os passeios a pé, seguidos pelas viagens de automóvel foram os meios de transportes mais utilizados nestas atividades.

\section{GERAÇÃO DE VIAGENS}

A Tabela 1 apresenta as taxas médias e os modelos de geração de viagem desenvolvidos pelo ITE (2008) para as categorias "Hotel" e "Hotel Resort" para dias de semana, sábado, e domingo, no dia, na hora de pico da via pela manhã e pela tarde e na hora de pico do hotel, de manhã e de tarde. As variáveis utilizadas foram o número de quartos, o número de quartos ocupados e o número de empregados. Apresenta-se também o número de empreendimentos utilizados na elaboração 
dos modelos, a distribuição direcional e para a regressão o valor de $\mathrm{R}^{2}$.

Na Tabela 2 são apresentadas as taxas de geração de viagens desenvolvidas para os hotéis do centro de Florianópolis, por Goldner e Inocêncio (2007). A Tabela 3 apresenta as taxas para os hotéis de praia.

Nestas tabelas observam-se os volumes de veículos entrando, saindo e total por dia e taxas calculadas, respectivamente, em função do número de quartos, do número de empregados, da área total construída (dividida por $100 \mathrm{~m}^{2}$ ) e do número de vagas de estacionamentos do hotel.

Da mesma maneira, observam-se na Tabela 4 e na
Tabela 5 os modelos de regressão linear simples elaborados por Goldner e Inocêncio (2007) a partir dos mesmos valores e tendo como variáveis independentes as mesmas já citadas. Neste caso apresenta-se também o valor de $\mathrm{R}^{2}$ e da estatística "t-student”, com o respectivo nível de significância.

Em relação aos estudos de Ackeret e Hosea (1992) e de Rowe et al (2002), os modelos não estão apresentados, pois os hotéis-cassino têm características distintas dos demais, dificultando uma análise comparativa com a realidade brasileira.

Tabela 1. Taxas e modelos de geração de viagens para hotéis - ITE (2008)

\begin{tabular}{|c|c|c|c|c|c|c|c|}
\hline Tipo & Período & Variável & $\begin{array}{c}N^{\circ} \text { de } \\
\text { estudos }\end{array}$ & $\begin{array}{c}\text { Distribuição Di- } \\
\text { recional }\end{array}$ & $\begin{array}{l}\text { Taxa } \\
\text { média }\end{array}$ & Equação/modelo & $R^{2}$ \\
\hline Hotel & Dia da semana & $\begin{array}{c}\text { Quartos } \\
\text { ocupados }\end{array}$ & 4 & $\begin{array}{c}50 \% \text { entrando/ } \\
50 \% \text { saindo }\end{array}$ & 8,92 & & \\
\hline Hotel & Sábado & $\begin{array}{c}\text { Quartos } \\
\text { ocupados }\end{array}$ & 3 & $\begin{array}{c}50 \% \text { entrando/ } \\
50 \% \text { saindo }\end{array}$ & 10,50 & & \\
\hline Hotel & Domingo & $\begin{array}{c}\text { Quartos } \\
\text { ocupados }\end{array}$ & 3 & $\begin{array}{c}50 \% \text { entrando/ } \\
50 \% \text { saindo }\end{array}$ & 8,48 & & \\
\hline Hotel & dia da semana & Quartos & 10 & $\begin{array}{c}50 \% \text { entrando/ } \\
50 \% \text { saindo }\end{array}$ & 8,17 & $\mathrm{~T}=8,95(\mathrm{X})-373,16$ & 0,98 \\
\hline Hotel & Sábado & Quartos & 8 & $\begin{array}{c}50 \% \text { entrando/ } \\
50 \% \text { saindo }\end{array}$ & 8,19 & $\mathrm{~T}=9,62(\mathrm{X})-294,56$ & 0,93 \\
\hline Hotel & Domingo & Quartos & 8 & $\begin{array}{c}50 \% \text { entrando/ } \\
50 \% \text { saindo }\end{array}$ & 5,95 & $\operatorname{Ln}(T)=1,34 \operatorname{Ln}(X)-0,11$ & 0,94 \\
\hline Hotel & Dia da semana & Empregados & 5 & $\begin{array}{c}50 \% \text { entrando/ } \\
50 \% \text { saindo }\end{array}$ & 14,34 & $\operatorname{Ln}(T)=1,36 \operatorname{Ln}(X)+0,96$ & 0,54 \\
\hline Hotel & Sábado & Empregados & 8 & $\begin{array}{c}50 \% \text { entrando/ } \\
50 \% \text { saindo }\end{array}$ & 12,27 & $\operatorname{Ln}(T)=0,80 \operatorname{Ln}(X)+3,47$ & 0,57 \\
\hline Hotel & Domingo & Empregados & 8 & $\begin{array}{c}50 \% \text { entrando/ } \\
50 \% \text { saindo }\end{array}$ & 8,92 & $\mathrm{~T}=9,15(\mathrm{X})-32,07$ & 0,67 \\
\hline
\end{tabular}

Tabela 2. Taxas de viagens para os hotéis do centro - Goldner e Inocêncio (2007)

\begin{tabular}{|c|c|c|c|c|c|c|c|c|c|c|c|c|c|c|c|c|c|c|c|c|}
\hline \multirow{2}{*}{$\begin{array}{c}N^{o} \\
\text { Hotel }\end{array}$} & \multirow{2}{*}{ Nome do Hotel } & \multicolumn{3}{|c|}{$N^{\circ}$ de viagens diárias } & \multirow{2}{*}{$\begin{array}{c}X 1 \\
N^{o} d e \\
q u a r t o s\end{array}$} & \multirow{2}{*}{$\begin{array}{c}\text { Taxa } \\
X 1 / \\
\text { entrando }\end{array}$} & \multirow{2}{*}{\begin{tabular}{c|} 
Taxa \\
$X 1 /$ \\
saindo
\end{tabular}} & \multirow{2}{*}{$\begin{array}{c}\text { Taxa } \\
\text { X1/ } \\
\text { total }\end{array}$} & \multirow{2}{*}{$\begin{array}{c}X 2 \\
N^{o} \text { de } \\
\text { Empre- } \\
\text { gados }\end{array}$} & \multirow{2}{*}{$\begin{array}{c}\text { Taxa } \\
X 2 / \\
\text { entrando }\end{array}$} & \multirow{2}{*}{$\begin{array}{c}\text { Taxa } \\
X 2 / \\
\text { saindo }\end{array}$} & \multirow{2}{*}{$\begin{array}{c}\text { Taxa } \\
X 2 / \\
\text { total }\end{array}$} & \multirow{2}{*}{$\begin{array}{c}\mathrm{X3} \\
\text { Área Total } \\
\text { Cons } / 100 \mathrm{~m}^{2}\end{array}$} & \multirow{2}{*}{$\begin{array}{c}\text { Taxa } \\
\text { X3/ } \\
\text { entrando }\end{array}$} & \multirow{2}{*}{$\begin{array}{c}\text { Taxa } \\
\text { X3/ } \\
\text { saindo }\end{array}$} & \multirow{2}{*}{$\begin{array}{c}\text { Taxa } \\
\text { X3/ } \\
\text { total }\end{array}$} & \multirow{2}{*}{$\begin{array}{c}X 4 \\
\text { Vagas } \\
\text { Estac. }\end{array}$} & \multirow{2}{*}{$\begin{array}{c}\text { Taxa } \\
X 4 / \\
\text { entrando }\end{array}$} & \multirow{2}{*}{$\begin{array}{c}\text { Taxa } \\
X 4 / \\
\text { saindo }\end{array}$} & \multirow{2}{*}{$\begin{array}{c}\text { Taxa } \\
X 4 / \\
\text { total }\end{array}$} \\
\hline & & Entrando & Saindo & Total & & & & & & & & & & & & & & & & \\
\hline 1 & Majestic Palace & 173 & 174 & 347 & 275 & 0,63 & 0,63 & 1,26 & 142 & 1,22 & 1,23 & 2,44 & Nobtido & Nobtido & Nobtido & Nobtido & 295 & 0,59 & 0,59 & 1,18 \\
\hline 2 & Hotel Ibis & 319 & 354 & 673 & 198 & 1,61 & 1,79 & 3,40 & 39 & 8,18 & 9,08 & 17,26 & Nobtido & Nobtido & Nobtido & Nobtido & 120 & 2,66 & 2,95 & 5,61 \\
\hline 3 & Bristol Castelmar & 123 & 129 & 252 & 192 & 0,64 & 0,67 & 1,31 & 96 & 1,28 & 1,34 & 2,63 & 200,00 & 0,62 & 0,65 & 1,26 & 72 & 1,71 & 1,79 & 3,50 \\
\hline 4 & InterCity & 122 & 125 & 247 & 122 & 1,00 & 1,02 & 2,02 & 68 & 1,79 & 1,84 & 3,63 & 70,35 & 1,73 & 1,78 & 3,51 & 40 & 3,05 & 3,13 & 6,18 \\
\hline 5 & Hotel Faial & 101 & 101 & 202 & 119 & 0,85 & 0,85 & 1,70 & 70 & 1,44 & 1,44 & 2,89 & 200,00 & 0,51 & 0,51 & 1,01 & 45 & 2,24 & 2,24 & 4,49 \\
\hline 6 & Baía Norte Palace & 155 & 143 & 298 & 108 & 1,44 & 1,32 & 2,76 & 55 & 2,82 & 2,60 & 5,42 & 53,00 & 2,92 & 2,70 & 5,62 & 41 & 3,78 & 3,49 & 7,27 \\
\hline 7 & Valerim Plaza & 158 & 169 & 327 & 105 & 1,50 & 1,61 & 3,11 & 55 & 2,87 & 3,07 & 5,95 & 57,30 & 2,76 & 2,95 & 5,71 & 50 & 3,16 & 3,38 & 6,54 \\
\hline 8 & Florianópolis Palace & 45 & 49 & 94 & 99 & 0,45 & 0,49 & 0,95 & 55 & 0,82 & 0,89 & 1,71 & 68,00 & 0,66 & 0,72 & 1,38 & Nobtido & Nobtido & Nobtido & Nobtido \\
\hline 9 & Blue Tree Towers & 179 & \begin{tabular}{|l|l}
207 \\
\end{tabular} & 386 & 95 & 1,88 & 2,18 & 4,06 & 40 & 4,48 & 5,18 & 9,65 & 74,58 & 2,40 & 2,78 & 5,18 & 35 & 5,11 & 5,91 & 11,03 \\
\hline \multicolumn{5}{|c|}{ Média } & 145,89 & 1,11 & 1,17 & 2,29 & 68,89 & 2,77 & 2,96 & 5,73 & 103,32 & 1,66 & 1,72 & 3,38 & 57,57 & 3,10 & 3,27 & 6,37 \\
\hline \multicolumn{5}{|c|}{ Desvio Padrão } & 61,95 & 0,51 & 0,58 & 1,09 & 32,38 & 2,33 & 2,64 & 4,97 & 66,46 & 1,06 & 1,10 & 2,15 & 30,04 & 1,11 & 1,32 & 1,39 \\
\hline
\end{tabular}

Tabela 3. Taxas de viagens para os hotéis das praias - Goldner e Inocêncio (2007)

\begin{tabular}{|c|c|c|c|c|c|c|c|c|c|c|c|c|c|c|c|c|c|c|c|c|}
\hline \multirow{2}{*}{$\begin{array}{l}N^{\circ} \\
\text { Hotel }\end{array}$} & \multirow{2}{*}{ Nome do Hotel } & \multicolumn{3}{|c|}{$N^{\circ}$ de viagens diárias } & \multirow{2}{*}{$\begin{array}{c}X 1 \\
N^{o} d e \\
\text { quartos }\end{array}$} & \multirow{2}{*}{\begin{tabular}{|c|} 
Taxa \\
$X 1 /$ \\
entrando
\end{tabular}} & \multirow{2}{*}{\begin{tabular}{|c} 
Taxa \\
$X 1 /$ \\
saindo
\end{tabular}} & \multirow{2}{*}{$\begin{array}{c}\text { Taxa } \\
X 1 / \\
\text { total }\end{array}$} & \multirow{2}{*}{$\begin{array}{c}X 2 \\
N^{o} d e \\
\text { Empre- } \\
\text { gados }\end{array}$} & \multirow{2}{*}{$\begin{array}{c}\text { Taxa } \\
\text { X2/ } \\
\text { entrando }\end{array}$} & \multirow{2}{*}{$\begin{array}{c}\text { Taxa } \\
X 2 / \\
\text { saindo }\end{array}$} & \multirow{2}{*}{$\begin{array}{l}\text { Taxa } \\
X 2 / \\
\text { total }\end{array}$} & \multirow{2}{*}{$\begin{array}{c}X 3 \\
\text { Área Total } \\
\text { Cons } / 100 \mathbf{m}^{2}\end{array}$} & \multirow{2}{*}{\begin{tabular}{|c|} 
Taxa \\
$X 3 /$ \\
entrando
\end{tabular}} & \multirow{2}{*}{$\begin{array}{c}\text { Taxa } \\
\text { X3/ } \\
\text { saindo }\end{array}$} & \multirow{2}{*}{$\begin{array}{c}\text { Taxa } \\
X 3 / \\
\text { total }\end{array}$} & \multirow{2}{*}{$\begin{array}{c}X 4 \\
\text { Vagas } \\
\text { Estac. }\end{array}$} & \multirow{2}{*}{$\begin{array}{c}\text { Taxa } \\
X 4 / \\
\text { entrando }\end{array}$} & \multirow{2}{*}{$\begin{array}{c}\text { Taxa } \\
X 4 / \\
\text { saindo }\end{array}$} & \multirow{2}{*}{$\begin{array}{c}\text { Taxa } \\
X 4 / \\
\text { total }\end{array}$} \\
\hline & & Entrando & Saindo & Total & & & & & & & & & & & & & & & & \\
\hline 1 & Jurerê Beach Village & 156 & 180 & 336 & 120 & 1,30 & 1,50 & 2,80 & 90 & 1,73 & 2,00 & 3,73 & 231,31 & 0,67 & 0,78 & 1,45 & 200 & 0,78 & 0,90 & 1,68 \\
\hline 2 & $\begin{array}{l}\text { Praia Mole Park } \\
\text { Hotel }\end{array}$ & 72 & 81 & 153 & 93 & 0,77 & 0,87 & 1,65 & 70 & 1,03 & 1,16 & 2,19 & 800,00 & 0,09 & 0,10 & 0,19 & 100 & 0,72 & 0,81 & 1,53 \\
\hline 3 & $\begin{array}{l}\text { Hotel C. N. Ponta das } \\
\text { Canas }\end{array}$ & 59 & 48 & 107 & 55 & 1,07 & 0,87 & 1,95 & 48 & 1,23 & 1,00 & 2,23 & 24,81 & 2,38 & 1,93 & 4,31 & 20 & 2,95 & 2,40 & 5,35 \\
\hline 4 & \begin{tabular}{|l|} 
Canas vieiras Praia \\
Hotel
\end{tabular} & 25 & 25 & 50 & 54 & 0,46 & 0,46 & 0,93 & 30 & 0,83 & 0,83 & 1,67 & 40,00 & 0,63 & 0,63 & 1,25 & 28 & 0,89 & 0,89 & 1,79 \\
\hline 5 & $\begin{array}{l}\text { Hotel Costa Norte } \\
\text { Ingleses }\end{array}$ & 74 & 67 & 141 & 52 & 1,42 & 1,29 & 2,71 & 50 & 1,48 & 1,34 & 2,82 & Nobtido & Nobtido & Nobtido & Nobtido & Nobtido & Nobtido & Nobtido & Nobtido \\
\hline 6 & $\begin{array}{l}\begin{array}{l}\text { Joaquina Beach Park } \\
\text { Hotel }\end{array} \\
\end{array}$ & 56 & 61 & 117 & 32 & 1,75 & 1,91 & 3,66 & 10 & 5,60 & 6,10 & 11,70 & 14,80 & 3,78 & 4,12 & 7,90 & 26 & 2,15 & 2,58 & 4,50 \\
\hline \multicolumn{5}{|c|}{ Média } & 67,7 & 1,13 & 1,15 & 2,28 & 49,67 & 1,98 & 2,07 & 4,06 & 222,18 & 1,51 & 1,51 & 3,02 & 74,8 & 1,50 & 1,52 & 2,97 \\
\hline \multicolumn{5}{|c|}{ Desvio Padrão } & 32,39 & 0,46 & 0,52 & 0,97 & 28,30 & 1,80 & 2,01 & 3,81 & 335,08 & 1,53 & 1,53 & 1,59 & 77,3 & 1,00 & 0,89 & 1,81 \\
\hline
\end{tabular}


Tabela 4. Modelos de regressão linear - hotéis do centro - Goldner e Inocêncio (2007)

\begin{tabular}{|c|c|c|c|c|c|c|c|}
\hline Variável "Y" & Variável “X” & Equação & $\begin{array}{l}N^{o} . d e \\
\text { casos }\end{array}$ & $R^{2}$ & $\begin{array}{c}\text { Erro } \\
\text { Padrão } \\
\end{array}$ & $\begin{array}{c}\text { Teste "t" } \\
\text { student }\end{array}$ & Signif. \\
\hline V entra & $\mathrm{N}^{\mathrm{o}}$ quartos & $Y=0,972 X$ & 9 & 0,824 & 74,96 & 6,114 & 0,000 \\
\hline V sai & $\mathrm{N}^{\circ}$ quartos & $Y=1,025 X$ & 9 & 0,798 & 85,93 & 5,620 & 0,000 \\
\hline V entra + sai & $\mathrm{N}^{\mathrm{o}}$ quartos & $Y=1,997 X$ & 9 & 0,811 & 160,59 & 5,861 & 0,000 \\
\hline V entra & $\mathrm{N}^{\mathrm{o}}$ empregados & $\mathrm{Y}=1,781 \mathrm{X}$ & 9 & 0,636 & 107,70 & 3,739 & 0,006 \\
\hline V sai & $\mathrm{N}^{\circ}$ empregados & $Y=1,850 X$ & 9 & 0,599 & 121,03 & 3,458 & 0,009 \\
\hline V entra + sai & $\mathrm{N}^{\circ}$ empregados & $Y=3,633 X$ & 9 & 0,617 & 228,54 & 3,593 & 0,007 \\
\hline V entra & $\mathrm{N}^{\mathrm{o}}$ vagas-estac. & $Y=1,108 X$ & 8 & 0,561 & 125,92 & 2,992 & 0,020 \\
\hline V sai & $\mathrm{N}^{\circ}$ vagas-estac. & $Y=1,161 X$ & 8 & 0,537 & 138,45 & 2,850 & 0,025 \\
\hline V entra + sai & $\mathrm{N}^{\mathrm{o}}$ vagas-estac. & $Y=2,268 X$ & 8 & 0,549 & 264,13 & 2,921 & 0,022 \\
\hline V entra & Área cons/100 & $Y=1,068 X$ & 9 & 0,481 & 128,55 & 2,726 & 0,026 \\
\hline V sai & Área cons/100 & $\mathrm{Y}=1,129 \mathrm{X}$ & 9 & 0,469 & 139,31 & 2,658 & 0,029 \\
\hline V entra + sai & Área cons/100 & $Y=2,197 X$ & 9 & 0,475 & 267,62 & 2,693 & 0,027 \\
\hline
\end{tabular}

Tabela 5. Modelos de regressão linear - hotéis das praias - Goldner e Inocêncio (2007)

\begin{tabular}{|c|c|c|c|c|c|c|c|}
\hline Variável “Y” & Variável “ $X "$ & Equação & $\begin{array}{l}N^{o} . d e \\
\text { Casos }\end{array}$ & $R^{2}$ & $\begin{array}{c}\text { Erro } \\
\text { Padrão }\end{array}$ & $\begin{array}{c}\text { Teste " } t \text { " } \\
\text { student }\end{array}$ & Signif. \\
\hline V entra & $\mathrm{N}^{\mathrm{o}}$ quartos & $Y=1,090 X$ & 6 & 0,920 & 33,07 & 7,568 & 0,001 \\
\hline V sai & $\mathrm{N}^{\mathrm{o}}$ quartos & $Y=1,179 X$ & 6 & 0,907 & 30,52 & 6,985 & 0,001 \\
\hline V entra + sai & $\mathrm{N}^{\mathrm{o}}$ quartos & $Y=2,268 X$ & 6 & 0,916 & 55,68 & 7,368 & 0,001 \\
\hline V entra & $\mathrm{N}^{\mathrm{o}}$ empregados & $Y=1,432 X$ & 6 & 0,913 & 27,18 & 7,224 & 0,001 \\
\hline V sai & $\mathrm{N}^{\mathrm{o}}$ empregados & $Y=1,536 X$ & 6 & 0,889 & 33,86 & 6,221 & 0,002 \\
\hline V entra + sai & $\mathrm{N}^{\mathrm{o}}$ empregados & $Y=2,968 X$ & 6 & 0,901 & 60,34 & 6,745 & 0,001 \\
\hline V entra & $\mathrm{N}^{\mathrm{o}}$ vagas-estac. & $Y=0,805 X$ & 5 & 0,914 & 28,17 & 6,507 & 0,003 \\
\hline V sai & $\mathrm{N}^{\mathrm{o}}$ vagas-estac. & $Y=0,913 X$ & 5 & 0,948 & 24,42 & 8,512 & 0,001 \\
\hline V entra + sai & No vagas-estac. & $Y=1,718 X$ & 5 & 0,933 & 52,26 & 7,486 & 0,002 \\
\hline \multirow{2}{*}{ V entra } & \multirow{2}{*}{ Area cons/100 } & \multirow{2}{*}{$Y=40,518+0,489 X$} & \multirow{2}{*}{5} & \multirow{2}{*}{0,827} & \multirow{2}{*}{23,60} & $\mathrm{t} 1=2,944$ & 0,060 \\
\hline & & & & & & $\mathrm{t} 2=3,790$ & 0,032 \\
\hline \multirow{2}{*}{ V sai } & \multirow{2}{*}{ Area cons/100 } & \multirow{2}{*}{$Y=33,762+0,620 X$} & \multirow{2}{*}{5} & \multirow{2}{*}{0,886} & \multirow{2}{*}{23,45} & $\mathrm{t} 1=2,469$ & 0,090 \\
\hline & & & & & & $\mathrm{t} 2=4,835$ & 0,017 \\
\hline \multirow{2}{*}{ V entra + sai } & \multirow{2}{*}{ Area cons/100 } & \multirow{2}{*}{$Y=74,280+1,109 X$} & \multirow{2}{*}{5} & \multirow{2}{*}{0,864} & \multirow{2}{*}{46,43} & $\mathrm{t} 1=2,743$ & 0,071 \\
\hline & & & & & & $\mathrm{t} 2=4,369$ & 0,022 \\
\hline
\end{tabular}

\section{ANÁLISE COMPARATIVA DOS MODELOS}

\subsection{Dimensão Metodológica}

A compilação dos estudos existentes mostra a influência exercida pelos trabalhos do ITE na produção de pesquisas sobre o tema. O trabalho do ITE (2008) utiliza a regressão linear, bem como os demais. A exceção é o trabalho de Feitosa (2003) que realizou entrevistas para conhecer os padrões de viagens a hotéis residência na Cidade do Rio de Janeiro, Brasil, o mesmo acontecendo com Goldner et al para hotéis da cidade de Florianópolis. O ITE (2008) usou o modelo de regressão linear simples, Ackret e Hosea (1992) regressão linear simples e múltipla. Rowe et al (2002) aperfeiçoaram os estudos de Ackret e Hosea (1992), calibrando os modelos e classificando-os em dois tipos conforme a localização na cidade. Goldner e Inocêncio (2007) usaram a regressão linear.

As taxas e os modelos de geração de viagens foram desenvolvidos para diferentes tipos de hotéis. Assim, a análise comparativa ficará restrita aos modelos do ITE com os modelos desenvolvidos por Goldner e Inocêncio (2007). Quanto a categoria, considerar-se-á os "hotéis" da publicação do ITE e os "hotéis do centro" do estudo de Goldner e Inocêncio (2007).

Realizando-se uma simulação para um cenário de um hotel típico com 100 quartos e 47 empregados, através do cálculo do número de viagens diárias (Y) relacionadas com as variáveis: número de quartos (X1) e número de empregados (X2), utilizando-se os modelos do ITE para hotéis e os modelos propostos para hotéis do centro, obtiveram-se os valores apresentados nas tabelas 6 e 7, respectivamente. A definição dos valores de 100 quartos e 47 empregados foi realizada a partir das proporções encontradas nos hotéis do centro na amostra da cidade de Florianópolis.

Comparando-se as duas realidades, observa-se que os modelos do ITE apresentaram valores $259 \%$ maiores que os do modelo proposto, calculando-se através 
Tabela 6. Resultados da simulação total utilizando modelos do ITE (2008)

\begin{tabular}{|c|c|c|c|}
\hline \multicolumn{4}{|c|}{ Hotel-dia de semana } \\
\hline & Total & Entrando & Saindo \\
\hline \multicolumn{4}{|l|}{ Variável X1 = Número de quartos do hotel } \\
\hline Taxa Média = 8,17 & $\mathrm{Y}=817$ viagens $/$ dia & 408,5 viagens/dia & 408,5 viagens/dia \\
\hline Equação: $\mathrm{Y}=$ 8,95 (X1) - 373,16 & $\mathrm{Y}=522$ viagens $/$ dia & 261 viagens/dia & 261 viagens/dia \\
\hline \multicolumn{4}{|l|}{ Número de casos $=10$} \\
\hline \multicolumn{4}{|l|}{$\mathrm{R}^{2}=0,98$} \\
\hline & Total & Entrando & Saindo \\
\hline \multicolumn{4}{|l|}{ Variável X2 = Número de empregados } \\
\hline Taxa Média = 14,34 & $\mathrm{Y}=674$ viagens $/$ dia & 337 viagens/dia & 337 viagens/dia \\
\hline Equação: $\ln (\mathrm{Y})=1,36 \ln (\mathrm{X} 2)+0,96$ & $\mathrm{Y}=491$ viagens $/ \mathrm{dia}$ & 245,5 viagens/dia & 245,5 viagens/dia \\
\hline \multicolumn{4}{|l|}{ Número de casos $=5$} \\
\hline $\mathrm{R}^{2}=0,54$ & & & \\
\hline
\end{tabular}

Tabela 7. Resultados da simulação utilizando os modelos de Goldner e Inocêncio (2007)

\begin{tabular}{|c|c|c|c|}
\hline \multicolumn{4}{|c|}{ Hotel do centro - dia de semana } \\
\hline & Total & Entrando & Saindo \\
\hline \multicolumn{4}{|c|}{ Variável X1 = Número de quartos do hotel } \\
\hline Taxa Média = 2,29 & $\mathrm{Y}=229$ viagens $/$ dia & 111 viagens/dia & 117 viagens/dia \\
\hline Equação: Y = 1,997 X1 & $Y=200$ viagens $/$ dia & 97,5 viagens/dia & 102,5 viagens/dia \\
\hline \multicolumn{4}{|l|}{ Número de casos $=9$} \\
\hline \multicolumn{4}{|l|}{$\mathrm{R}^{2}=0,811$} \\
\hline & Total & Entrando & Saindo \\
\hline \multicolumn{4}{|c|}{ Variável X2 = Número de empregados } \\
\hline Taxa Média = 5,73 & $Y=269$ viagens $/$ dia & 130 viagens/dia & 139 viagens/dia \\
\hline Equação: $Y=3,633$ X2 & $\mathrm{Y}=171$ viagens $/$ dia & 84 viagens/dia & 87 viagens/dia \\
\hline \multicolumn{4}{|l|}{ Número de casos $=9$} \\
\hline $\mathrm{R}^{2}=0,617$ & & & \\
\hline
\end{tabular}

das taxas de viagens médias, e 163\% maiores calculando-se através da equação, isto para variável número de quartos. Para variável número de empregados os valores são $151 \%$ e $187 \%$ maiores, respectivamente.

Nas Figuras 1 e 2, a seguir, apresentam-se as retas derivadas dos modelos de regressão elaborados pelo ITE (2008) e Goldner e Inocêncio (2007), para as variáveis número de quartos e número de empregados.

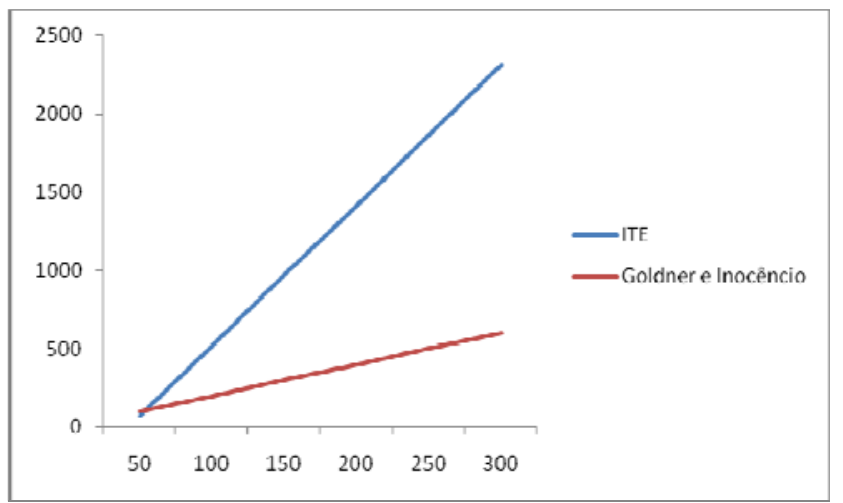

Figura 1. Comparação Quantitativa dos Modelos (variável número de quartos)

\subsection{Padrão do PGV}

No que diz respeito ao Padrão do PGV que embasa os métodos, a diferença está relacionada ao tipo de hotel. O ITE (2008) apresenta hotéis com ou sem suítes, hotéis de negócios, motéis e resort hotéis. Ackret e Hosea (1992) bem como Rowe et al (2002) estudam os hotéis-cassinos, Feitosa (2003) os hotéis-residências e Goldner et al (2006) os hotéis tradicionais.

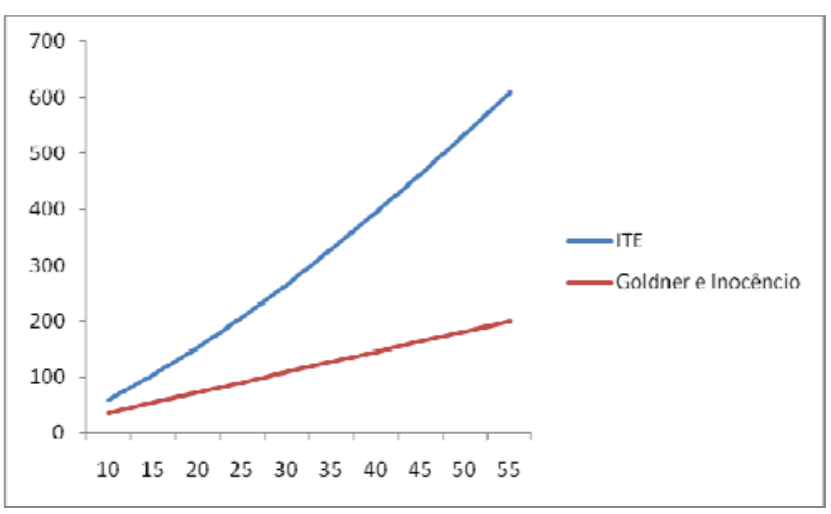

Figura 2. Comparação Quantitativa dos Modelos (variável número de empregados) 


\subsection{Dimensão Espacial}

Para a análise da localização dos elementos que compõem a amostra pesquisada por cada modelo, poderse-ia fazer a distinção entre os hotéis-cassinos dentro da cidade de Las Vegas em Ackret e Hosea (1992) e Rowe et al (2002), os hotéis de praia e do centro da cidade de Florianópolis de Goldner et al (2006) e os hotéis-residências usados por Feitosa (2003) na zona sul e Barra da Tijuca, devido às características turísticas dessas duas regiões da cidade do Rio de Janeiro.

\subsection{Dimensão Temporal}

Com relação ao Dia de Projeto, o modelo do ITE faz estimativas para um dia típico da semana, para sábado e domingo; Feitosa (2003) para os dias da semana e Goldner et al (2007), devido as flutuações da demanda na Cidade de Florianópolis, levantou informações nos sábados e terças-feiras , separadamente, no verão e nos meses típicos.

\subsection{Padrão de Viagens}

Em todos os trabalhos há um destaque para o aspecto qualitativo. Nos estudos do ITE (2008) as viagens têm como propósito reuniões, turismo e turismo de negócios; no estudo de Ackret e Hosea (1992) e Rowe et al (2002) turismo de jogo; Goldner et al (2006) turismo e negócios e Feitosa (2003) trabalho.

\section{CONCLUSÕES}

O presente trabalho analisou diferentes bibliografias que estudaram os hotéis sobre a ótica da Engenharia de Tráfego e do planejamento, tanto no Brasil como no exterior. Pode-se observar que a bibliografia é escassa e que os trabalhos foram desenvolvidos para diferentes categorias de hotéis.

O foco principal de investigação foram as taxas e modelos de geração de viagens desenvolvidos pelos diferentes autores. Como informação complementar apresentou-se alguns estudos sobre os padrões de viagem.

De maneira geral observa-se que os autores utilizam modelos de regressão linear, simples ou múltiplos, relacionando o número de viagens (entrada, saída, total) com variáveis de fácil obtenção como o número de quartos, o número de empregados, e a área construída do hotel.

Devido à diversidade de categorias de hotéis estudados, tornou-se difícil realizar uma análise comparativa dos resultados obtidos com diferentes modelos. A comparação viável entre a categoria hotéis dos modelos do ITE com a categoria hotéis do centro de Florianópolis do estudo de Goldner e Inocêncio (2007) mostrou que o modelo americano superestima o número de viagens em relação ao modelo brasileiro. Naturalmente, para emprego deste modelo, deve-se levar em consideração a realidade em que eles se aplicam, sendo possível utilizá-los para estudos em Florianópolis ou cidades com características similares.

Espera-se que o estudo realizado para Florianópolis represente um estímulo aos pesquisadores do país, de modo que outras realidades sejam analisadas, com proposição de modelos adequados. Ao trabalho de compilação dos modelos de geração de viagens para hotéis aliam-se outros estudos, realizados para diferentes PGV's, desenvolvidos por pesquisadores da rede Ibero-Americana de Estudos em Pólos Geradores de Viagens, representando assim mais uma contribuição para o fortalecimento desta rede e desta linha de pesquisa no país.

\section{REFERÊNCIAS BIBLIOGRÁFICAS}

Ackeret, K.W; Hosea, R. C. (1992) Trip Generation Rates for Las Vegas Area Hotel-Casinos. ITE Journal, May, 33-37.

CET/SP - Companhia de Engenharia de Tráfego (1983) Pólos Geradores de Tráfego. Boletim Técnico, São Paulo, n. 32.

CET/SP - Companhia de Engenharia de Tráfego (2001) Pólos Geradores de Tráfego II. Boletim Técnico, São Paulo, n. 36. Código de Trânsito Brasileiro, lei n ${ }^{\circ} 9503$ de 23 de Setembro de 1997, Diário Oficial da União, Brasília DF.

Conceição, I. (1984) Shopping center: desenvolvimento, localização e impacto no sistema viário. Dissertação deMestrado - Programa de Engenharia de Transportes, COPPE/Universidade Federal do Rio de Janeiro, RJ.

DENATRAN - Departamento Nacional de Trânsito (2001) Manual de procedimentos para o tratamento de Pólos Geradores de Tráfego. Brasília. Estatuto das Cidades, lei 10.257 de 10 de Julho de 2001, Diário oficial da União, Brasília, DF.

Feitosa, T. C. G.; Balassiano, R. (2003) Gerenciamento da mobilidade em Pólos Geradores de Tráfego: análise de hotéis-residência no município do Rio de Janeiro. Anais do XVII ANPET- Congresso de Pesquisa e Ensino em Transportes, Rio de Janeiro.

Feitosa, T. C. G. (2003) Gerenciamento da mobilidade em Pólos Geradores de Tráfego: análise de hotéis- residência no município do Rio de Janeiro. Dissertação de Mestrado. Programa de Engenharia de Transportes, COPPE/Universidade Federal do Rio de Janeiro, RJ.

Giustina, C. D. (2005) Uma análise da demanda de shopping centers de Porto Alegre a partir de dados provenientes de pesquisas domiciliares de origem e destino. Dissertação de Mestrado. Programa de Pós-Graduação em Engenharia de Produção, Universidade Federal do Rio Grande do Sul, Porto Alegre.

Goldner, L. G; Ventura, T. S. e Inocêncio, P. (2006) Elaboração de taxas de geração de viagens e parâmetros do estacionamento e do meiofio de embarque/desembarque para hotéis. Anais do XX ANPETCongresso de Pesquisa e Ensino em Transportes, Brasília, DF.

Goldner, L. G; Inocêncio, P. (2007) Elaboração de modelos de geração de viagens terrestres para hotéis. Anais do XXI ANPET- Congresso de Pesquisa e Ensino em Transportes, Rio de Janeiro,RJ.

Goldner, L.G.; Goldner, N.; Pedrozo, D. E. (2005) Parâmetros para dimensionamento dos estacionamentos de automóveis em aeroportos brasileiros. Anais do XIX ANPET- Congresso de Pesquisa e Ensino em Transportes, vol.1, p.804, Recife.

Goldner, L. G. (1994) Uma metodologia de avaliação de impactos de shopping centers sobre o sistema viário urbano. Tese de Doutorado. Programa de Engenharia de Transportes, COPPE/Universidade Federal do Rio de Janeiro, RJ

Goldner, L. G.; Silva, R. H. (1996) Uma análise dos supermercados como Pólos Geradores de Tráfego. X ANPET-Congresso de Pesquisa e Ensino em Transportes, Brasília.

Grando, L. A (1986) Interferência dos Pólos Geradores de Tráfego no sistema viário: análise e contribuição metodológica para shopping centers. Dissertação de Mestrado. Programa de Engenharia de Transportes, COPPE/Universidade Federal do Rio de Janeiro, RJ.

ITE - Institute of Transportation Engineers (1991) Traffic access and impacts studies for site development. Washington D.C.

ITE - Institute of Transportation Engineers (2003) Trip Generation, 7th edition: an ITE informational report. 
ITE - Institute of Transportation Engineers (2008) Trip Generation, 8th edition. Washington D.C.

ITE - Institute of Transportation Engineers (2001) Trip Generation Handbook - an ITE recommended practice, publicação n.RP-028A. Washington D.C.

Keefer, L. E. (1966) Urban travel patterns for airports, shopping centres, and industrial plants. National cooperative highway research program. Report 24, Highway Research Board.

Kikuchi, S.; Gupta, A.; Felsen, M.; Brockenbrough, T.W. (2006) Trip atraction characteristics of neighhood-and community-level shopping centers. TRB Annual Meeting, CD-ROM.

Kneib, E. C. (2004) Caracterização de empreendimentos geradores de viagens: Contribuição conceitual à análise de seus impactos no uso, ocupação e valorização do solo urbano. Dissertação de Mestrado. Departamento de Engenharia Civil e Ambiental, Universidade de Brasília, Brasília.

Nunes, J. L.; Jacques, M. A. P. (2005) Caracterização dos padrões de viagens em instituições de ensino Superior. Anais do XIX ANPET Congresso de Pesquisa e Ensino em Transportes, vol.1, p.780, Recife.

Peyrebrune, J. C. (1996) Trip generation característics of shopping centers. ITE Journal, Washington D.C., v. 66, n. 6, p. 46.

Portugal, L. S.; Goldner, L. G. (2003) Estudo de pólos geradores de tráfego e de seus impactos nos sistemas viários e de transportes. 1. ed. Rio de Janeiro: Editora Edgard Blücher LTDA, 322p.

Rede Ibero-Americana de estudos em Pólos Geradores de Viagens. Disponível em: <http://redpgv.coppe.ufrj.br>. Acesso em: Abril de 2010.

Rowe, C. D.; Kaseko, M. S.; Ackeret, K. W. (2002) Recalibration of Trip Generation Model for Las Vegas Hotel/Casinos. ITE Journal, May, p. 26-33.

Saab, W. G. L. e Gimenez, L.C.P. (2001) Flats, apart-hotéis ou hotéisresidência: Caracterização e desempenho no Brasil e no município de São Paulo. BNDS setorial, Rio de Janeiro, Setembro, n. 14, p. 137-157.

Silva, L. R. (2006) Estudo das características da área de influência dos pólos geradores de tráfego e sua relação com a geração de viagens - um estudo de caso nos supermercados e hipermercados. Dissertação de Mestrado. PPGT, Universidade de Brasília, Brasília.

Silveira, I. T (1991) Análise de Pólos Geradores de Tráfego segundo sua classificação, área de influência e padrões de viagem. Dissertação de Mestrado. Programa de Engenharia de Transportes, COPPE/Universidade Federal do Rio de Janeiro, RJ.

United States Department of Transportation; ITE - Institute of Transportation Engineers (1985) Site impact traffic evaluation (SITE). Relatório final. Washington. 